\title{
Design of a New Energy Absorber Based on the Coulomb Friction to Protect Structures Against Impact Loads
}

\author{
D. Karayel*, G. Atali And S.S. OzKan \\ Sakarya University, Department of Mechatronics Engineering, 54100 Sakarya, Turkey
}

\begin{abstract}
This study aims to design a new energy absorber (damper) to protect structures against impact loads and against sudden transition from resonance region. It is an adaptation of interference fit process in machine elements for designing of damper based on the principles of Coulomb friction. There are a lot of investigations of dampers based on Coulomb friction. In this study, pre-stress is obtained by interference fit. It is different from other studies and is original in this respect. There is a contact pressure between the shaft and the hub in the interference fit joints and frictional force occurs with the beginning of the relative motion in the axial direction. Thus, the damaging energy is absorbed from the system. The quantity of damping ratio depends directly on the tolerances of the shaft and the hub and can be easily adjusted. This is another advantage of the friction damper with interference fit compared to similar dry friction dampers.
\end{abstract}

DOI: 10.12693/APhysPolA.134.109

PACS/topics: energy absorber, coulomb friction, protecting structures, impact loads

\section{Introduction}

Protection of structures against impact loads has great importance. Therefore, any structure is generally designed so that it can act by staying out of resonance region. Sometimes it may be inevitable that some structure would operate in the resonance region for a period of time and any structure may be influenced by the impact loads, such as earthquakes.

Structural damages may occur is such cases and this can cause the loss of life and property if the released energy can not be removed from the structure. Therefore, passive and active dampers have been developed. However, the issue still remains and is under investigation of scientists.

Salehghaffari et al. [1] have performed an experimental investigation of two new structural design solutions with the aim of improving crash-worthiness characteristics of cylindrical metal tubes. They prepared and compressed several specimens of each developed design with various geometric parameters. They have shown the significant efficiency of the presented design methods in improving crash-worthiness characteristics, and investigated collapse modes, of circular tubes under axial loading.

Azimi and Asgari [2] investigated single and double wall structures for crash-worthiness and introduced a novel system with better energy absorption and crushing characteristics under both axial and oblique loading. They developed a new structure and this structure improved the absorbed energy under oblique loading.

Dong and Lakes have studied an advanced damper with negative structural stiffness elements [3]. Kathiresan and Manisekar [4] have studied the axial crush be-

*corresponding author; e-mail: dKarayel@sakarya.edu.tr haviour and energy absorption characteristics of aluminium and E-glass/epoxy over-wrapped aluminium conical frusta under low velocity impact loading. They simulated the low velocity impact tests with the help of finite element analysis, using the ABAQUS software, in order to predict and to compare the crash-worthiness of each category of specimen model with experimental results. In their study, they saw that the obtained crash-worthiness test results and collapse behaviour of finite element analysis are in good agreement with the experimental results.

Mohammadpour and Chakherlou [5] have presented a framework of combined kinematic and isotropic hardening for finite deformation cyclic plasticity. Their study is based on an interference fitted joint using a large deformation Chaboche type combined isotropic-kinematic hardening law and mortar contact method. Results of their study show that changes in stress are steep in the initial cycles. They also suggested the idea that one can assess fatigue life based on further cycle results.

Qiu and Zhou [6] proposed an analytical method to calculate the contact pressure and stress between multilayer thick-walled cylinders with multi-contact pairs and temperature-raising effect. They have shown that the analytical method presented in their study can reduce the complexity of problems for multi-layer thick-walled cylinders and improve the computational efficiency.

Mualla and Belev have studied the dynamic response of single-storey steel frames equipped with a novel friction damper device. A new friction damper device which was developed for seismic protection of structures has been described and evaluated experimentally and numerically in their study [7].

In addition to basic studies, dampers are also very effective against earthquakes. In [8-10] researchers studied friction dampers for reducing the seismic responses and their seismic performance during an earthquake.

Schmitz et al. [11] studied the stiffness and damping of shrink fit tool holder connection for frequency response 
prediction in milling. In their studies the receptance coupling substructure analysis (RCSA) approach for joining parts of the tool and holder with reduced connection is described, the RCSA equation for the entire tool holdershaft assembly is given and the finite element modeling for the reduced connection is carried out.

The friction damper has an important role in vibration damping. However, the occurrence of pressure on the friction surfaces has always been a problem and researchers generally focus on this part of the problem. In this study, the pressure on the contact surfaces is obtained by the dimensional difference between the shaft and the hub. In such case it is very easy to obtain pressure and the pressure distribution over the cylindrical surface is homogeneous. The study is quite different from other studies in this respects.

\section{Interference fits and fitting tolerances}

An interference fit is also known as a press fit or friction fit and it is used as a fastening method for hubs and shafts in mechanical assembly process. In press fits, the shaft is compressed and the hub is expanded which is similar to phenomena in a pressurized cylinder. Hence, interference fit calculations are made similar to those of pressurized cylinders. The interference fit process is shown in Fig. 1.

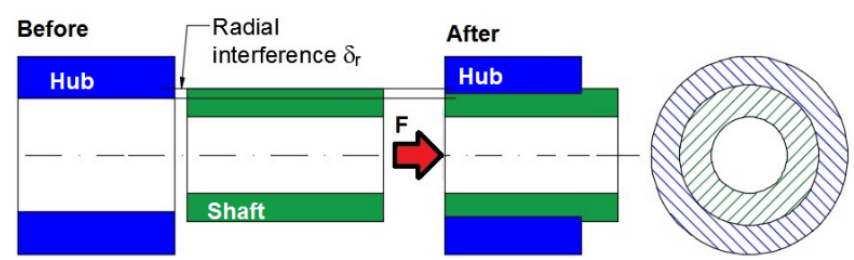

Fig. 1. Interference fits.

At the beginning, let us denote by $\delta$ the radial interference, which is the difference between the $r_{2}$ values of the shaft and the hub. In the following equations the shaft and the hub have been described as cylinder 1 and cylinder 2, respectively (Fig. 2).
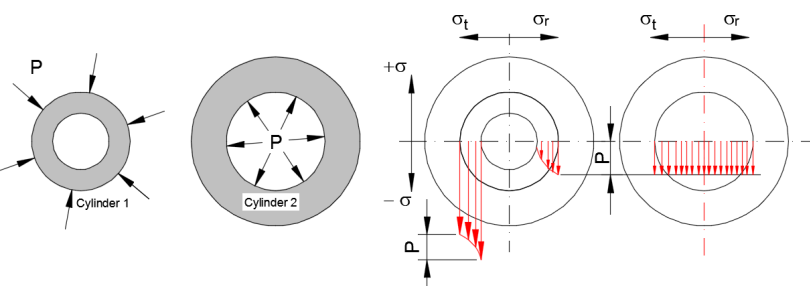

Fig. 2. Stress distribution in the inner and outer cylinders.

The radial and tangential stresses can be respectively written as follows

$$
\sigma_{r}=C_{1}+\frac{C_{2}}{r^{2}}, \quad \sigma_{\theta}=C_{1}-\frac{C_{2}}{r^{2}} .
$$

Boundary conditions for cylinder 1 are

$$
\begin{aligned}
& r=r_{1} \rightarrow \sigma_{r}=0, \\
& r=r_{2} \rightarrow \sigma_{r}=-P .
\end{aligned}
$$

Boundary conditions for cylinder 2 are

$$
\begin{aligned}
& r=r_{2} \rightarrow \sigma_{r}=-P, \\
& r=r_{3} \rightarrow \sigma_{r}=0 .
\end{aligned}
$$

The general interference equation is written as follows

$$
U=\frac{r}{E}\left[(1-v) C_{1}-(1+v) \frac{C_{2}}{r^{2}}\right] .
$$

Coefficients $C_{1}$ and $C_{2}$ can be found using the presented boundary conditions. If these coefficients are substituted into the equations above, then for cylinder 1 (shaft):

$$
\begin{aligned}
& \sigma_{r \mathrm{I}}=-P \frac{r_{2}^{2}}{r_{2}^{2}-r_{1}^{2}}\left(1-\frac{r_{1}^{2}}{r^{2}}\right), \\
& \sigma_{\theta \mathrm{I}}=-P \frac{r_{2}^{2}}{r_{2}^{2}-r_{1}^{2}}\left(1+\frac{r_{1}^{2}}{r^{2}}\right), \\
& U_{\mathrm{I}}=\frac{P r_{2}^{2} r}{\left(r_{2}^{2}-r_{1}^{2}\right) E}\left[(1-v)+(1+v) \frac{r_{1}^{2}}{r^{2}}\right] .
\end{aligned}
$$

For cylinder 2 (hub):

$$
\begin{aligned}
\sigma_{r \mathrm{II}} & =P \frac{r_{2}^{2}}{r_{3}^{2}-r_{2}^{2}}\left(1-\frac{r_{3}^{2}}{r^{2}}\right), \\
\sigma_{\theta \mathrm{II}} & =\frac{P r_{2}^{2} r}{r_{3}^{2}-r_{2}^{2}}\left(1+\frac{r_{3}^{2}}{r^{2}}\right), \\
U_{\mathrm{II}} & =\frac{P r_{2}^{2} r}{\left(r_{3}^{2}-r_{2}^{2}\right) E}\left[(1-v)+(1+v) \frac{r_{3}^{2}}{r^{2}}\right] .
\end{aligned}
$$

The total radial interference is

$$
U=\left|\left(U_{\mathrm{I}}\right)_{r=r_{2}}\right|+\left|\left(U_{\mathrm{II}}\right)_{r=r_{2}}\right|=\delta \text {. }
$$

If the ratios of radii are denoted as

$$
\begin{aligned}
& Q_{\mathrm{H}}=r_{2} / r_{3} \text { and } Q_{\mathrm{S}}=r_{1} / r_{2}, \\
& U=p d\left[\frac{1}{E_{\mathrm{H}}}\left(\frac{1+Q_{\mathrm{H}}^{2}}{1-Q_{\mathrm{H}}^{2}}+\frac{1}{v_{\mathrm{H}}}\right)+\frac{1}{E_{\mathrm{S}}}\left(\frac{1+Q_{\mathrm{S}}^{2}}{1-Q_{\mathrm{S}}^{2}}-\frac{1}{v_{\mathrm{S}}}\right)\right] .
\end{aligned}
$$

Here $E_{\mathrm{H}}$ is elastic modulus of the hub, $E_{\mathrm{S}}$ is elastic modulus of the shaft, $v_{\mathrm{H}}$ is Poisson's ratio for the hub and $v_{\mathrm{S}}$ is Poisson's ratio for the shaft.

If the hub and the shaft are of the same material with the same $E$ and the shaft is solid, then

$$
\begin{aligned}
& Q_{\mathrm{S}}=r_{1} / r_{2}=0, \\
& E_{\mathrm{H}}=E_{\mathrm{S}}=E, \\
& v_{\mathrm{H}}=v_{\mathrm{S}}=v, \\
& 2 r=d
\end{aligned}
$$

are written and if the interference equation is rearranged, 


$$
U=\frac{2 p d}{E\left(1-Q_{\mathrm{H}}^{2}\right)} .
$$

The interface pressure is required in order to find the damping force in the axial direction. The radial interference $U$ can be found from the fitting tolerance. The interface pressure and the fitting (damping) force can be calculated as

$$
\begin{aligned}
& P=\frac{U E\left(1-Q_{\mathrm{H}}^{2}\right)}{2 d}, \\
& \sum F_{\mathrm{d}}=\pi d l p \mu .
\end{aligned}
$$

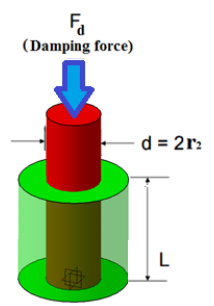

Damping force per unit length can be found using the following formula,

$F_{\mathrm{d}}=\pi d p \mu$.

\section{Damping analysis of interference fits}

In this study the damping effect of interference fit process has been analyzed using analytical and numerical methods.

\subsection{Analytical method}

This method is based on the formula of damping force per unit length. For this method the process parameters shown in Table I have been selected and different values for damping force have been obtained for different combinations of process parameters. The changes in damping forces per unit length obtained for each solution are graphically presented in Fig. 3 .

TABLE I

Process parameters of interference fit application.

\begin{tabular}{c|c|c|c|c}
\hline \hline Sample & $d$ & $D$ & Material & Fitting tolerance \\
\hline 1 & 60 & 70 & $\mathrm{St} 42$ & $\mathrm{H} 7 / \mathrm{n} 6$ \\
2 & 60 & 70 & $\mathrm{St} 42$ & $\mathrm{H} 7 / \mathrm{r} 6$ \\
3 & 60 & 80 & $\mathrm{St} 42$ & $\mathrm{H} 7 / \mathrm{n} 6$ \\
4 & 60 & 80 & $\mathrm{St} 42$ & $\mathrm{H} 7 / \mathrm{r} 6$ \\
5 & 60 & 90 & $\mathrm{St} 42$ & $\mathrm{H} 7 / \mathrm{n} 6$ \\
6 & 60 & 90 & $\mathrm{St} 42$ & $\mathrm{H} 7 / \mathrm{r} 6$
\end{tabular}

\subsection{Finite element simulation}

The hub and the shaft have been designed and modeled. The modeled hub and shaft are presented in Fig. 4. The commercial finite element code, ABAQUS, has been used to carry out the simulation. Both the hub and the shaft have been considered as made of deformable materials. Both, the hub and the shaft are made of steel for which the Young modulus is $2.1 \times 10^{5} \mathrm{GPa}$ and the Poisson ratio is 0.3 .

7410 elements and 36795 nodes have been used in the FEM model. Element type was set to 3D stress

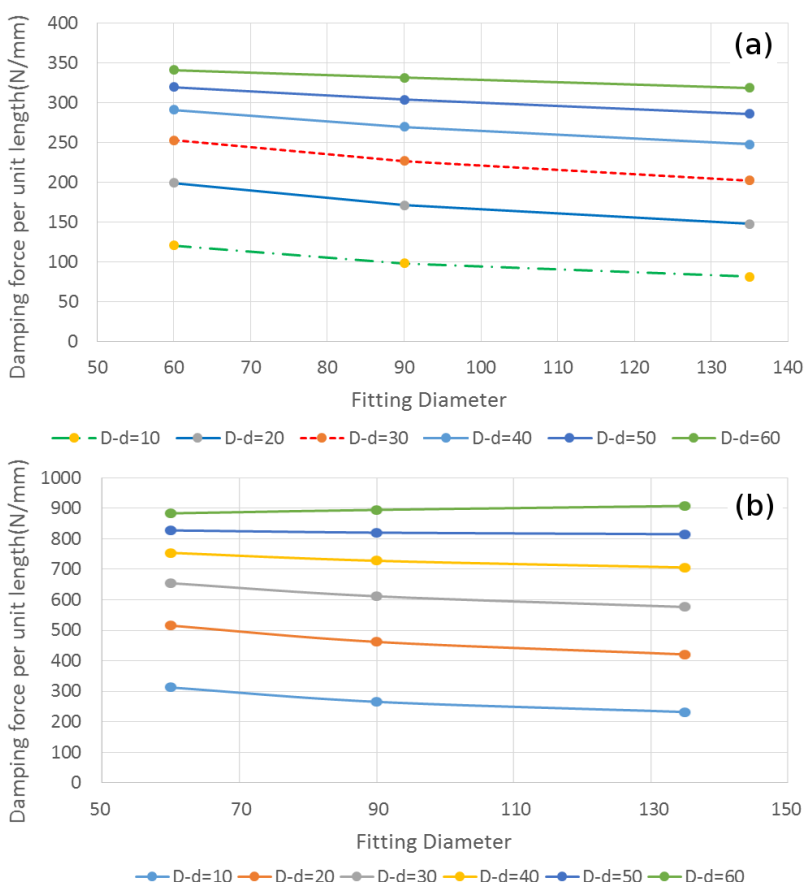

Fig. 3. Plot of damping force versus fitting diameter for cases of (a) H7/n6, (b) H7/r6.
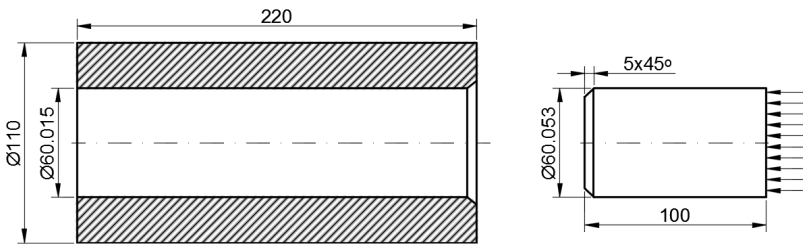

Fig. 4. The dimensions of the hub (a), the shaft (b).

(C3D20R quadratic linear) element. The friction coefficient between the contact surfaces was set to 0.15. A fine mesh has been applied on the contact surfaces and other regions. The numerical solution was performed in 11 steps. The distribution of the calculated stress is shown in Fig. 5.
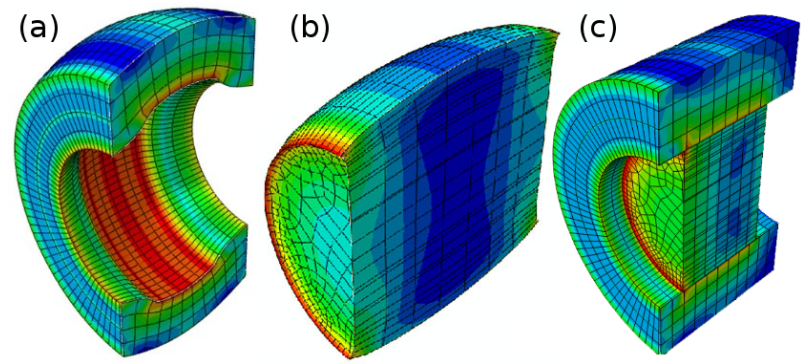

Fig. 5. The results of numerical simulation; (a) hub, (b) shaft, (c) hub and shaft. 


\section{Design of energy absorber}

The proposed friction damper based on interference fit principle can be used in many areas against earthquakes, crashing, dropping, shocks and impacts. This article is our first study on the subject and it is intended to analyze the usability of interference fit process in damping elements.

The analysis carried out in the previous sections has shown very promising results. In this section a damper is considered to be used in steel structure against the earthquake motion. This damper is mounted on the structure as shown in Fig. 6.
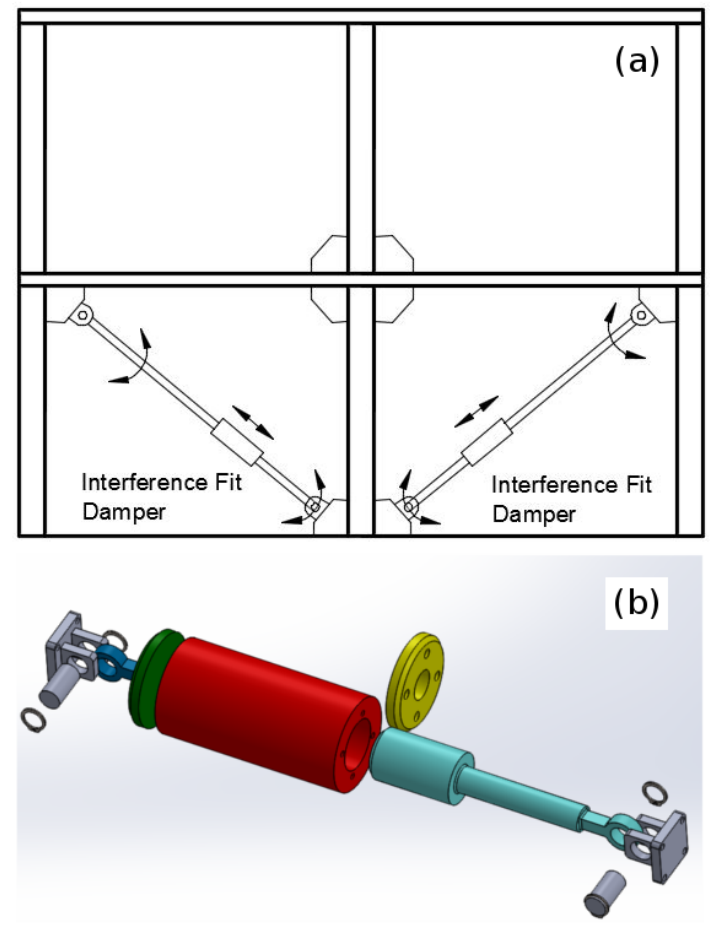

Fig. 6. The position of the damper inside the steel structure (a) and the construction of the damper (b).

The damper shaft moves forward and backward in the cylinder because of the lateral translations of the entire system and thus, damping occurs due to the frictional force between the contact surfaces. $\mathrm{H} 7 / \mathrm{r} 6$ has been used as ISO fitting tolerance in the developed damper. Damper parameters and geometric dimensions are the same as those used in previous analyzes. The designed damper consists of a cylinder, a piston, two covers and the parts of hinge joints.

\section{Conclusions}

Both analytical and numerical results clearly show that interference fit process is a very promising principle, which can be used for the damping of impacts and vibrations. It is very easy to achieve the required damping force if the fitting tolerances are selected correctly and a proper design is made. It is seen that if the fitting is too tight the damper will act like a solid material. However, if the fit is less tight the damper will not provide damping force. Both cases mean that the damper will not work correctly. Therefore, the interference fit needs to be adjusted very well according to degree of shock and impact.

A preliminary design of the interference damper has been realized using the obtained initial results. The interference damper can be easily adapted for many applications of shock and vibration damping. This is the first study and an optimization of the system has to be carried out. We expect that the developed absorber will contribute to structural dynamics and will have a wide area of use. The next study will be focused on the manufacturing of the designed damper, tests will be realized on a real system and the results will be compared with the results of numerical simulation.

\section{References}

[1] S. Salehghaffari, M. Tajdari, M. Panahi, F. Mokhtarnezhad, Thin-Walled Struct. 48, 379 (2010).

[2] M.B. Azimi, M. Asgari, Int. J. Mechanical Sci. 105, 253 (2016).

[3] L. Dong, R.S. Lakes, Smart Mater. Struct. $\mathbf{2 1}$ 075026 (2012).

[4] M. Kathiresan, K. Manisekar, Composite Struct. 136 , 86 (2016).

[5] A. Mohammadpour, T.N. Chakherlou, Int. J. Mechanical Sci. 106, 297 (2016).

[6] J. Qiu, M. Zhou, Appl. Sci. 6, 167 (2016).

[7] I.H. Mualla, B. Belev, Eng. Struct. 24, 365 (2002).

[8] A.V. Bhaskararao, R.S. Jangid, Eng. Struct. 28, 690 (2006).

[9] K.-W. Min, J.-Y. Seong, J. Kim, Eng. Struct. 32, 3539 (2010)

[10] A. Heysami, Curr. World Environ. 10, 1002 (2015).

[11] T.L. Schmitz, K. Powell, D. Won, G.S. Duncan, W.G. Sawyer, J.C. Ziegert, Int. J. Machine Tools and Manufacture 47, 1368 (2007). 